\title{
Rechtliche Fragen und Fakten zu Managed Care
}

\author{
Lässt sich die Budgetmitverantwortung - Kern des Managed-Care-Modells - über- \\ haupt mit geltendem Recht vereinbaren? Diese Frage hat der Verein für Ethik und \\ Medizin Schweiz in einem Gutachten klären lassen.
}

\section{Michel Romanens,}

Eduard Hafner, Flavian Kurth

Verein Ethik und Medizin Schweiz (VEMS)
1 Trivedi A, Moloo H, Mor V. Increased ambulatory care copayments and hospitalizations among the elderly. New Engl J Med. 2010;362:320-8.

2 http://physicianprofiling.ch/ KieserMC082011Final.pdf

Korrespondenz:

Dr. med. Michel Romanens

Verein Ethik und Medizin

Schweiz

Ziegelfeldstrasse 1

CH-4600 Olten

info[at]kardiolab.ch

www.vems.ch

\section{Wer Fragen stellt, fliegt raus}

Mit Hochdruck wurde die Managed-Care-Vorlage durchs Parlament gepeitscht und ist nun nur noch durch ein Referendum zu stoppen. Wer Fragen stellt, gilt als ewiggestriger Bremser, der einem Idyll des Arztberufes von einst nachträumt. Der Verein Ethik und Medizin Schweiz (VEMS) möchte in diese emotionale Diskussion ein paar Fakten einbringen. Das Problem der versteckten Rationierung innerhalb von Managed-Care-Modellen wurde bereits von verschiedener Seite thematisiert und ist nicht Gegenstand dieser Betrachtung. Ebenfalls kommt eine Studie zum Schluss, dass eine Erhöhung des Selbstbehalts längerfristig zu Mehrkosten führt, weil die Patienten - um Geld zu sparen - gesundheitliche Probleme vor sich herschieben und erst dann den Arzt aufsuchen, wenn die Behandlung überfällig, ergo kompliziert, ergo teuer ist [1]. Die Strategie, den Versicherten via erhöhtem Selbstbehalt das Managed-Care-Modell aufzuzwingen, wäre vor diesem Hintergrund noch einmal zu überdenken. Uns hat aber viel grundlegender interessiert, ob sich eine Budgetmitverantwortung, die ja der Kern des Managed-Care-Modells ist, überhaupt mit geltendem Recht vereinbaren lässt.

\section{Gesetzliche Grundlagen}

Die gesetzlichen Rahmenbedingungen zur Ausübung des Arztberufes sind im Strafrecht, im Obligationenrecht und insbesondere im Medizinalberufegesetz festgeschrieben. Wir wollten wissen, ob diese Gesetze durch separate Verträge zwischen Leistungserbringern und Krankenversicherern tangiert werden. Mit dieser Frage sind wir an PD Dr. iur. Ueli Kieser gelangt und haben ihn gebeten, abzuklären, inwieweit Budgetverantwortung bzw. Budgetmitverantwortung, beispielhaft abzuklären für Managed-Care-Modelle, mit existierendem Recht vereinbar ist. Gemäss der Auffassung des VEMS ist die Ethik in der Beziehung zwischen Arzt und Patient gesetzlich geschützt durch das Obligationenrecht OR 398 Abs. 2 (Treuepflichten) und OR 41 ff. (unerlaubte Handlung), durch das Medizinalberufegesetz MedBG Art. 40 ff. und durch das Krankenversicherungsgesetz, insbesondere Art. 56 ff. (die Behandlung hat wirksam, zweckmässig und wirtschaftlich zu sein, WZW-Regel). Das Gutachten von PD Dr. iur. Ueli Kieser [2] kommt denn auch zum Schluss, dass hier durchaus eine juristisch problema-

\section{Managed care: questions et faits juridiques}

Chargé par l'association «Verein Ethik und Medizin» d'analyser la question de la compatibilité de la coresponsabilité budgétaire prévue par le Managed care avec le droit existant, Ueli Kieser, privat-docent et docteur en droit, livre des résultats surprenants. Dans son expertise, il insiste tout particulièrement sur la question de la transparence: le médecin affilié à un réseau doit impérativement informer son patient des conventions qui le lient avec les assureurs. L'auteur a également passé au crible les conditionscadres légales et notamment la loi sur les professions médicales, qui interdit au médecin de réaliser un quelconque profit qui ne soit pas directement dans l'intérêt du patient. II en tire des conclusions tout aussi stupéfiantes: premièrement, le Parlement n'a pas suffisamment pris en compte les conditionscadres légales existantes dans ses délibérations sur le Managed care, et deuxièmement, la transmission des données n'est pas assez réglementée dans les modèles de soins intégrés. L'expertise d'U. Kieser permet désormais $d^{\prime} y$ voir plus clair sur cette tendance croissante à mélanger les intérêts des caisses et des patients. Une chose est sûre: le choix du traitement devrait reposer exclusivement sur l'intérêt du patient. Après tout, la loi l'exige.

tische Situation entsteht: «Insgesamt ergeben sich also auf die Beachtung von Art. 40 lit e MedBG grundlegende und ernsthafte Fragen, die bislang in der parlamentarischen Beratung nicht aufgegriffen und insoweit auch nicht beantwortet wurden.»

\section{Annahmen statt Fakten}

Wieso diese Fragen in der parlamentarischen Betrachtung nicht aufgegriffen wurden, wäre Gegenstand 


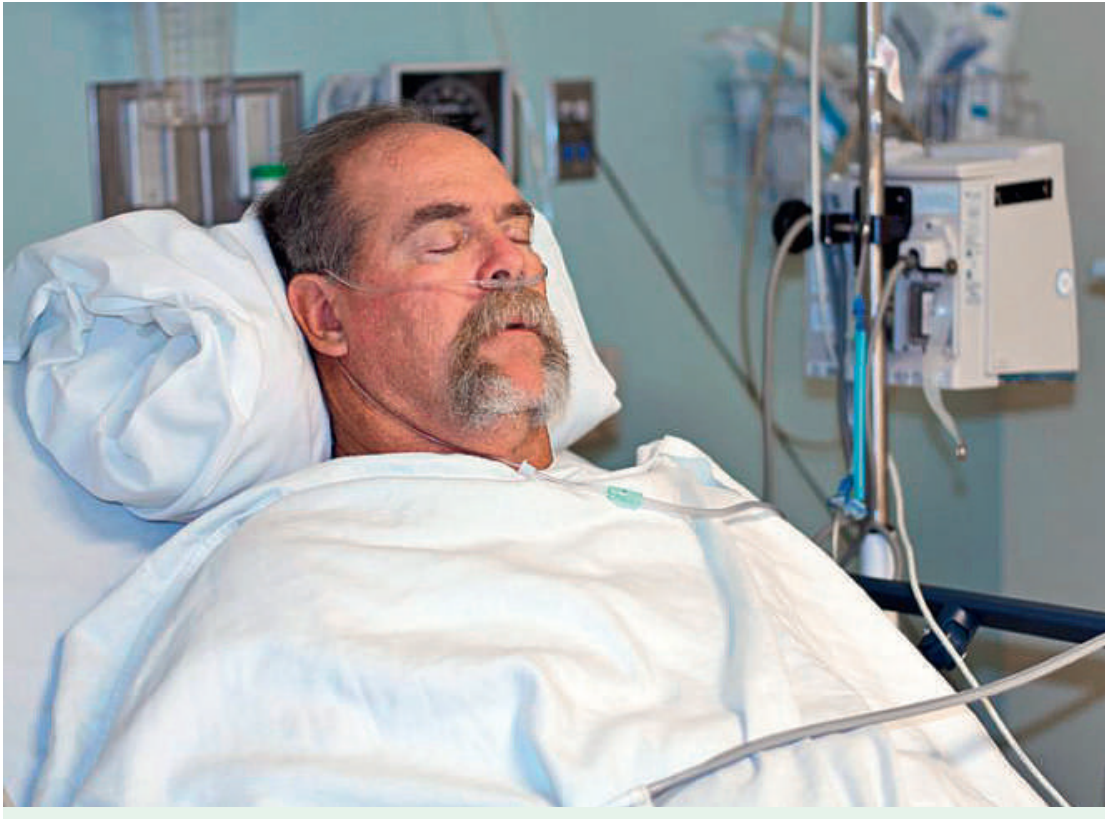

Der Arzt im Spannungsfeld der Ansprüche von Patient und Allgemeinheit, vertreten durch die Krankenkassen: Wie kann er beiden gerecht werden?
Überlegungen zu den Kosten anzustellen sind. Zuerst die Gesundheit, dann das Budget. Mit anderen Worten: zuerst die Anliegen des Patienten, dann die Anliegen der Kasse. Damit dies in der Praxis gewährleistet ist, fordert das Gutachten: «Die Patientin und der Patient müssen darüber informiert sein, dass der Arzt und die Ärztin in ein entsprechendes Netzwerk eingebunden sind und gegebenenfalls einen Verlust zu tragen haben oder am Gewinn beteiligt werden ... Es muss - ohne Einschränkung - sichergestellt sein, dass der Entscheid über die Behandlung ausschliesslich unter gesundheitsbezogenen Aspekten gefällt wird. Diesbezüglich werfen Managed-Care-Systeme erhebliche Fragen auf; diese müssen dahingehend beantwortet werden, dass zwingend Planken erforderlich sind, um die - gesundheitspolizeilich zu gewährleistenden - Interessen der Patientinnen und Patienten zu wahren.» Mit Blick auf die Grundsätze des Auftragsrechts zur Verhinderung von Korruption fordert das Gutachten «... dass bekannt ist, welche finanziellen Vorteile in welcher Form gewährt werden und wie die Verlustverteilung geordnet ist. Es ist zu berücksichtigen, dass der Arzt und die Ärztin grundsätzlich Vergünstigungen der versicherten Person oder dem Versicherer weiter zu geben haben.» Bezüglich Datenrecht wird festgehalten: «Die Übermittlung von Daten im Rahmen von Managed-Care-Systemen bedarf einer klaren, verhältnismässigen und zweckbezogenen Regelung.» Die juristische Forderung ist klar: wenn Budgetmitverantwortung, dann Transparenz betreffend Gewinne und Verluste als Resultat vertraglicher Vereinbarungen zwischen Arzt und Kasse. orliegt, die das beweisen oder nur schon nahelege würde. Dass bisher mit Managed-Care-Modellen Geld gespart wurde, hat seinen Hauptgrund in der Struktur der mit diesem Modell Versicherten. Dass dies auch für Chroniker, polimorbide und betagte Patienten der Fall ist, ist eine reine Annahme. Doch zurück zur rechtlichen Situation.

\section{Eindeutiges Vertragsverhältnis Arzt-Patient}

Das vollständige Gutachten von PD Dr. iur. Ueli Kieser kann auf www.physicianprofiling.ch heruntergeladen werden. Im Folgenden gehen wir auf die wesentlichen Punkte ein. Der Gutachter hält fest: «Der Arzt und die Ärztin haben die Interessen der Patientinnen und Patienten und deren Rechte unbedingt und ohne Vorbehalt zu wahren.» Zwischen Arzt und Patient herrscht ein Auftragsverhältnis, und der Arzt ist verpflichtet, ausschliesslich die Interessen der Patientinnen und Patienten zu wahren. Eine Verpflichtung gegenüber einer Drittpartei (Krankenkassen) tangiert dieses Verhältnis und birgt das Risiko der Korruption, insbesondere, wenn es, wie in der Budgetmitverantwortung von Managed-Care-Modellen, mit Gewinnoder Verlustbeteiligung verbunden ist.

\section{Kann man zwei Herren dienen?}

Das Gutachten kommt zum Schluss, dass der Hauptgrund einer medizinischen Massnahme ihre Wirksamkeit und Zweckmässigkeit sein muss und erst danach

\section{Wir haben immer schon zwei Herren gedient} Ist nun aber das von PD Dr. iur. Ueli Kieser Geforderte nicht immer schon Teil unserer Arbeit gewesen? Wir haben immer schon zwei Herren gedient, dem Patienten und der Allgemeinheit, vertreten durch die Krankenkassen. Ein Arzt, der jeden Simulanten krankschreibt, ist ebenso wenig ein guter Arzt wie einer, der nur die Ansprüche der Gesellschaft an das Individuum berücksichtigt und einem Kranken aus Kostenüberlegungen die Behandlung verweigert. Die Frage ist also nicht, ob wir zwei Herren dienen, sondern wie. Sind Managed-Care-Modelle in der Lage, uns bei der Abwägung von Wirksamkeit, Wirtschaftlichkeit und Zweckmässigkeit in unserer täglichen Arbeit zu helfen? Ihr Anreiz - nicht, wer mehr verschreibt, verdient mehr, sondern, wer weniger verschreibt - ist ein umgekehrter. Ist es deshalb der richtige? Vor dem Hintergrund all der bei der Ausarbeitung der Managed-Care-Vorlage offensichtlich übergangenen Fragen muss ernsthaft daran gezweifelt werden, dass dies überhaupt das Anliegen der Initianten war. Die Krankenkassen stellen in Frage, dass wir Ärzte das Wohl der Patienten vertreten. Gegenfrage: Vertreten die Krankenkassen das Wohl der Allgemeinheit? Vertritt die Managed-Care-Vorlage das Wohl der Allgemeinheit? Noch bleibt das Referendum! 